\title{
Concernment of various abutments for enhancing emergence profile in implant prosthodontics
}

\author{
Megha Sagar $^{1 *}$, Lakshya Vishnoi ${ }^{2}$, Varun Kumar $^{3}$, Himanshu Aeran ${ }^{4}$ \\ ${ }^{1} \mathrm{PG} 2{ }^{\text {nd }}$ Year, ${ }^{2}$ Intern, ${ }^{3}$ Professor, ${ }^{\mathbf{4}}$ Director Principal, Professor and Head, Dept. of Prosthodontics, ${ }^{\mathbf{1}, 3,4}$ Seema Dental College \& Hospital, \\ Rishikesh, Uttarakhand, ${ }^{2}$ KGMC, Lucknow, Uttar Pradesh, India \\ *Corresponding Author: Megha Sagar \\ Email: megha20sagar@gmail.com
}

\begin{abstract}
Implant abutments are artificial devices that are connected to the dental implants after the healing process is over. The abutments are used to attach a crown, bridge, or removable denture to the implant fixtures. Accurate prosthodontic rehabilitation is the key factor for providing the long-term success and the survival of osseointegrated implants. A precise fit of the implant is crucial since microgaps at the implant-abutment interface allow for microbial colonization, which can lead to peri-implant tissue inflammation. Polyetheretherketone (PEEK) has been used for academic investigations and commercialization of several dental devices, such as dental implants, abutments, healing caps, orthodontic braces, and most notably denture prosthetic frameworks.
\end{abstract}

Keywords: Implant Abutment, PEEK, CAD/CAM, Emergence profile.

\section{Introduction}

The prosthodontist's ability to conceptionalize the end result of treatment and to envision the final prosthesis in the space of the oral cavity is indeed a major clinical asset in planning and placing the prosthetic anchors in bone. The mind of the experienced prosthodontist is a virtual database; computing special orientation, leverage factors, loading forces, the biomechanics and physiology of the masticatory system and its relationship to occlusal harmony. Dental implants can help restore your smile if you have missing teeth. Once an implant is well integrated with the surrounding bone, its long-term success is heavily dependent on restorative biomechanical factors. Success depends on how the stresses imposed on a functioning implant or the prosthetic unit or units attached to the implant will be distributed to preserve the osseointegration of the implant. Implants are made of three parts, and together, these parts make a strong construction that allows you to function as you would with a full set of natural teeth. One of these parts is an abutment, and if you have an implant, you already have this part of the dental implant in your mouth.

\section{The tier system}

An abutment is a component that is intermediate between the implant and the restoration and is retained to the implant by a screw or locking taper. Abutments can usually be separated from the implant, but in some cases, they may form part of the implant itself. Additionally, not all implant restorations require abutments. In such situations, the crown is fabricated to be attached directly to the implant platform. The restorative part of the implant prosthesis is thus formed by an abutment providing the retention, support, stability and optimal position for the final restoration.

Most abutments are retained into the implant platform by a screw (Screw-retained) and the final crown can be screwretained directly onto the abutment (Three-Tier system) or directly onto the implant platform (Two-Tier system). Alternatively, the crown can be cemented as in a conventional crown and bridge protocol (Cement-retained) onto the abutment which is retained to the implant by a screw (Three-Tier system). Current implant systems enable the abutment to be locked or friction fit into the implant, without a screw or cement method of retention.

\section{Implant Abutment- Types}

Implant abutments are generally either custom made in a dental laboratory or prefabricated from implant manufacturers. ${ }^{1}$ Custom abutments are the fixture level impression of the implant platform that a dentist fabricates with the help of an impression coping. Custom abutments can be costly as they are commonly used in situations when prosthetic corrections with the standard prefabricated implant parts are not possible. Custom abutments are indicated in situations like:

1. Insufficient interocclusal restorative space

2. An angle correction problem greater than $15^{\circ}$

3. If the collar height needed is more than $1 \mathrm{~mm}$ greater than the largest collar height offered by the implant manufacturer.

4. If there is a need to replicate the original cross-sectional profile of the tooth in order to obtain an ideal emergence profile, and finally

5. If there is a need to splint three or more implants.

A prefabricated abutment is machine made by the manufacturer, and it may be selected directly by an implant or fixture level impression or it may be directly adapted to an existing platform and impressed as a conventional crown. Prefabricated abutments are less expensive and easy to use, but they do have some limitations and there are instances where such abutments are contraindicated. 


\section{Classification}

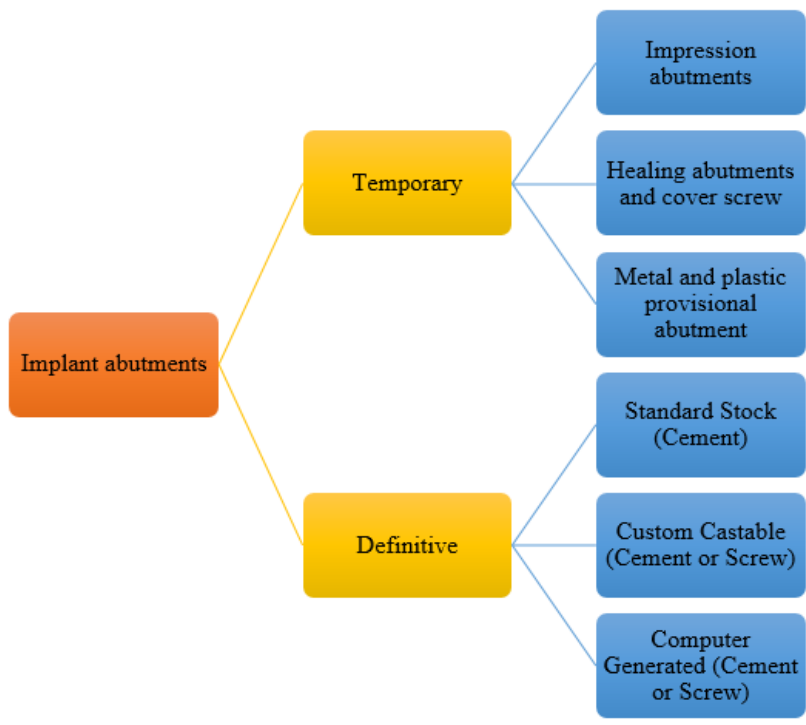

\section{A. Temporary Abutments}

Temporary abutments are usually machine premade produced in a stock prefabricated fashion. The clinician has the option to use them as it is or it can be customized as needed to establish tooth and gingival contours. Many of these abutments are modified to establish tissue contours especially in the esthetic region. These abutments help create the emergence profile, esthetic, phonetic boundaries, the position and the shade of the desired final restoration.

i. Impression Abutments: Open tray impression abutments are often called pick-up or direct copings. Closed tray impression abutments are often called Transfer or indirect copings.

ii. Healing Abutments: These abutments are used to cover the implant platform following surgical placement of the implant and to prevent tissue and bone growth into the implant body. It also helps to establish epithelization of the tissue and prevents influx of fluids from the oral cavity into the implant body. They can be used as a onestage or two-stage protocol. A one- stage surgical protocol allows the healing abutment to traverse the soft tissue in a transmucosal fashion and is exposed during the healing phase. This allows the dentist access directly to the implant platform without involving a second stage of surgery. Under two-stage protocol the entire implant platform is covered, sealed with a cover screw, under the soft tissue and requires a second surgery to expose the implant.

iii. Metal or plastic Abutment: These abutments are used following exposure of the implant platform and prior to the final restoration. They are used in the provisional phase and help to customize the form, shade, soft tissue profile and the occlusion prior to the definitive restoration. They can be metal titanium, ceramic zirconia or acrylic PEEK (polyetheretherketone). The abutments can also be engaging or non-engaging at their interfaces to the implant surface, which can provide the dentist with the option of using them in single or multi-unit situations. Following the natural gingival profile of the patient these can be fabricated in a standard stock round and anatomical profile. These can also be customized indirectly by the technician (laboratory) or directly by the dentist. ${ }^{2}$

\section{B. Definitive Abutments}

Permanent abutments are used for the final restoration and will remain in place definitively. The dentist has a choice during the final fabrication to use either a standard prefabricated, custom castable or computer-generated abutment.

i. Standard Stock Abutments: These are manufactured to standard sizes and can be used on both tissue level and bone level implants. They are designed to be userfriendly, to make impressions with ease, are the most economical and are typically used for cement retained restorations. They can be both straight and angled. They are best used for tissue level implants that are not in the esthetic zone.

The downside of stock implant abutments is that the tissue will conform to the shape of the implant abutment; if used in the esthetic zone, it is very difficult to for the ceramist to achieve optimal emergence contours and to have the tissue support be optimal relative to adjacent teeth. The other challenge is that the final margin placement of the crown cannot be precisely controlled - it is dependent on the depth of the implant and the height of the abutment.

ii. Custom/ Castable abutments: These abutments are milled to a precise position relative to the crest of the tissue; they can be used for either bone level or tissue level. There are many options for custom abutments, some are universal based upon a coping that is cemented, others are milled from a titanium or zirconia blank and some are simple tubes upon which a wax-up is performed. Used to correct extreme angulation problems or to deal with corrective abutment designs to accommodate proper coping and crown design where stock abutments cannot be used. They utilize UCLA (COBALT-CHROME), gold adapt, or cast to abutments for the casting process. ${ }^{2}$ Fig. 1

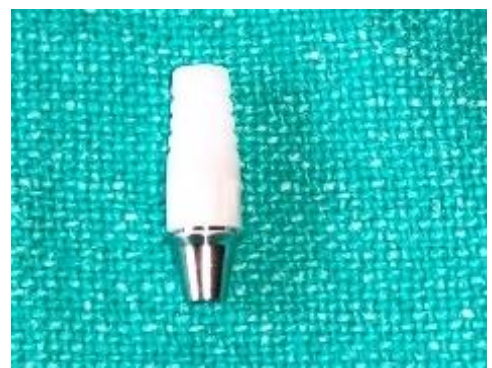

Fig. 1: Castable abutment 
iii. Computer Generated Abutments: CAD/CAM technology was incorporated into the production of implant abutments and frameworks in the 1980s. ${ }^{3}$ Custom abutments created with CAD/CAM technology are advantageous over both stock and laboratory processed custom abutments without any potential disadvantages. Fig. 2. The technician controls the abutment design using CAD software that incorporates parameters to assist him or her. The virtually designed abutment is electronically transferred to a CAM milling apparatus that creates the abutment from a block of the selected abutment material. Most of the inherent dimensional inaccuracies of waxing, investing, and casting are eliminated. Casting of titanium, in particular, is com- plex in a dental laboratory. CAD/CAM machining results in a homogenous mass of titanium with optimum material properties. ${ }^{4}$ Ideally, ceramic implant abutments should be machined from completely sintered blocks of material, but hard ceramic materials used for abutment fabrication can- not be efficiently machined with conventional grinding tools. Therefore, they are presently milled in the green body state before firing. ${ }^{5}$

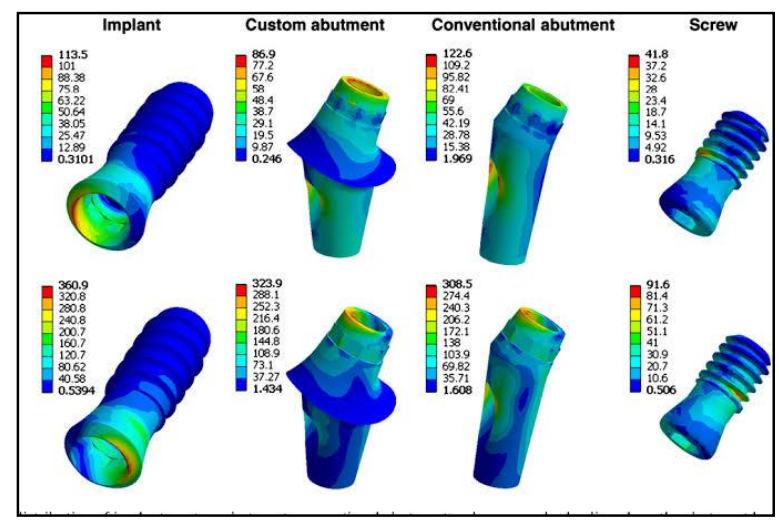

Fig. 2: Computer generated abutments

Currently available cad/cam abutment systems includes:

a) The Atlantis Abutment: (Atlantis Components, Inc, Cambridge, MA), milled in titanium alloy, has been commercially available since the early $1990 \mathrm{~s} .{ }^{6,7}$ Atlantis provides a second duplicate abutment to give dentists the option of placing a provisional crown on the first abutment and a definitive crown on the second. ${ }^{8}$ abutment may also be used as a laboratory die. Gold anodized coatings have been added to improve gingival tones and to impart more natural shades through allceramic restorations. Atlantis abutments are purported to have excellent marginal detail and anatomical gingival contours. ${ }^{9}$

b) Procera: Procera (Nobel Biocare, Yorba Linda, CA), initially developed for titanium and aluminum oxide copings for conventional crowns, ${ }^{10}$ has recently added implant abutments to their line of CAD/CAM components ${ }^{[1]}$ With this system, the abutment is virtually designed by the local laboratory using a Procera digital scanning system and software purchased from Nobel Biocare. The information is electronically transmitted to a Procera facility where the virtual abutment is milled and returned to the local laboratory. The dentist has the option to receive both a CAD/CAM abutment and CAD/CAM titanium or ceramic coping using this same system. Short-term results indicate that Procera ceramic abutments clinically perform as well as or better than other abutment types. ${ }^{12}$

c) Bella Tek ${ }^{\circledR}$ Endocde ${ }^{\circledR}$ (BIOMED 3i, Palm Beach Gardens, FL): is a robotic analog design. This system uses an impression of the healing abutment to design and fabricate the final abutment. These healing abutments come in a two-piece system which has notches on its occlusal surface which act as codes that the computer can scan and translate into implant abutment designs. ${ }^{[13]}$ The scanner can extract information about the position of the hex, the diameter of the implant platform, the collar height and then exact 3D placement of the implant. This then allows CAD and CAM fabrication.

d) Straumann ${ }^{\circledR}$ CARES ${ }^{\circledR}$ (Straumann, USA, Andover, MA): makes two-types of abutments: zirconium dioxide and titanium. In this system an implant level impression is made and then duplicate model of the master cast is fabricated. This is called the scan model and is made of a type of plaster that is scannable. ${ }^{14} \mathrm{~A}$ scan body is also attached to the implant analog prior to the scan to delineate implant position. The custom abutment is then designed CAD on screen to the contours needed and transmitted to the Straumann center. Etkon is another system that uses the same principles of waxing up onto a plastic cylinder which is then removed and scanned.

\section{Implant Abutment Materials}

A wide variety of abutment materials are available on the dental implant market. The clinicians must understand the biologic response to each material, as well as the best indication for using each of the different types. The most commonly used implant abutment materials are: Titanium (machined, polished, laser-lok), surgical grade stainless steel, cast gold, PEEK (Polyether ether ketone)

a) Titanium: Titanium is the only element that offers the unique combination of strength, light weight, and biocompatibility, as well as being extremely durable and strong. Titanium has high corrosion resistant and the highest strength to weight ratio of any known element. Commercially pure (CP) titanium is widely utilized for medical applications. The mechanical properties of $\mathrm{CP}$ titanium are influenced by small additions of oxygen and iron. By careful control of these additions, the various grades of CP titanium are produced to give properties suited to different applications. CP titanium with the lowest oxygen and iron levels makes the most formable grade of material; while progressively higher oxygen content results in higher strength levels. Titanium abutments come either with a silver gold color coating 
The gold color coating over the surface of the abutment is called titanium nitride. The titanium nitride (TiN; sometimes known as "Tinite," "TiNite," or "TiN") coating is created by a plasma coating process in which titanium and nitrogen ions are combined with TiN, and then molecularly bonded with the titanium substrate of the abutment. Titanium nitride is an extremely hard ceramic material, often used as a coating over the titanium component to not only improve the substrate's surface properties but also to achieve a warm, esthetic tone under the gingiva because of its gold shaded hue. Generally, the TiN coating covers the entire abutment except for the contact area between the abutment/implant and screw/abutment. This type of titanium abutment is ideal for esthetically challenging cases with thin soft tissue or when using an all-ceramic crown. In most applications the TiN coating is less than 5 micrometers $(0.00020$ inches) thick. This coating is only meaningful with CAD/CAM milled abutments where the abutment is not adjusted. Pre- fabricated abutments are adjusted and generally will lose any strength added by the nitrates following the abutment adjustment.

Titanium alloy (Ti-6Al-4V, Ti6Al4V, or Ti-6-4): Also called grade 5 titanium. Titanium alloy contains $6 \%$ aluminum, $4 \%$ vanadium, $0.25 \%$ (maximum) iron, $0.2 \%$ (maximum) oxygen, and the remainder titanium. Ti-6Al-4V alloy is significantly stronger than commercially pure titanium and offers better tensile strength and fracture resistance. Because of titanium's unique physical properties, titanium abutments are the first choice for posterior implants. These abutments are available as prefabricated stock or CAD/CAM milled customized abutments. ${ }^{15}$ Fig. 3

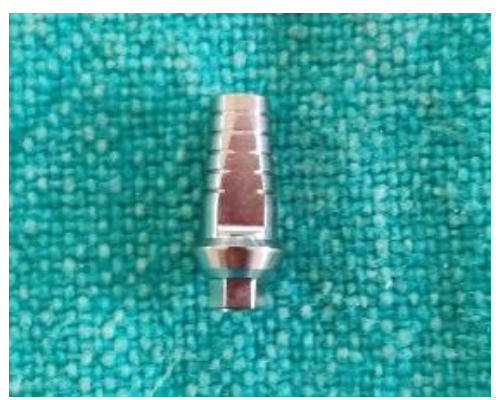

Fig. 3: Titanium implant abutment

b) Surgical Grade Stainless Steel: It is a specific type of stainless steel used in medical applications, and includes alloying elements of chromium, nickel, and molybdenum. The chromium gives the metal its scratch resistance and corrosion resistance. The nickel provides a smooth and polished finish. The molybdenum gives greater hardness and helps maintain a cutting edge. Stainless steel is easy to clean and sterilize, strong, and corrosion resistant. Surgical grade stainless steel can be used for temporary implant abutments but is not an ideal material of choice for permanent implant abutment since nickel/chrome/molybdenum alloys has immune system reaction to nickel.

c) Cast Gold: Recognizing the limitations of early "stock abutments" and developed a castable abutment called a
UCLA abutment. This abutment is comprised of a machined-fit gold alloy base that fits to the corresponding implant head, combined with a plastic sleeve which can be cut, modified, and added to with wax prior to casting into gold. Cast gold abutments were used to fabricate implant- level, custom-cast restorations that provided subgingival margins for esthetics, reduced height for vertical occlusal clearance, and/or custom angles. Generally, a plastic UCLA abutment is waxed up and customized to an ideal geometry and shape. After investing, the wax and plastic UCLA are burned out of the pattern following the lost wax process. When molten alloy is cast into the investment mold, the gold base component of the UCLA abutment is incorporated into the casting and provides a machined interface that precisely fits the implant. The gold base is fabricated from a non-oxidizing alloy that promotes chemical adhesion of the cast alloy, but does not permit the adhesion of porcelain. ${ }^{15}$

1. Gold specs: consists of $60-65 \%$ gold, $20-25 \%$ palladium, $19 \%$ platinum, and $1 \%$ iridium (not a ceramic alloy).

2. Melting range: Solid, $1400^{\circ} \mathrm{C}$; liquid, $1490^{\circ} \mathrm{C}$.

3. Recommended casting alloys: High palladium or high noble porcelain fusing alloys or type III or type IV high noble dental alloys.

Cast UCLA-type abutments lost popularity and have been replaced by CAD/CAM- milled or prefabricated options. However, the casting technique is an option for the design and fabrication of overdentures retained by telescopic crowns.

d) Polyether Ether Ketone (PEEK): PEEK has become the most popular material for fabricating temporary abutment. Fig. 4. It is a beige or white colored organic polymer and a semi crystalline thermo- plastic with excellent mechanical and chemical resistance properties. The Young's modulus is $3.6 \mathrm{GPa}$ and $\mathrm{MPa}$. PEEK has a glass transition temperature at around $143^{\circ} \mathrm{C}$ and melts at around $343^{\circ} \mathrm{C}$ $\left(662^{\circ} \mathrm{F}\right)$. It is highly resistant to thermal degradation as well as attack by both organic elements and moist environments. These robust properties have made PEEK an ideal material for temporary abutments. ${ }^{16}$ Furthermore, the oral microbial flora attachment to PEEK abutments is comparable to those made of titanium, zirconia and polymethylmethacrylate. ${ }^{17} \mathrm{~A}$ close match of elastic moduli of bone and PEEK surface reduces the stress shielding effects and encourage bone remodeling. Hence, PEEK could prove to be a viable alternative to titanium in constructing implant abutments.

Koutouzis et al, in 2011, provided a human prospective study comparing titanium and PEEK healing abutments. It was concluded that after 3 months there was not a significant difference between the two materials in terms of soft and hard tissue response. The response was measured in terms of plaque, bleeding on probing, and gingival and crestal bone height. ${ }^{18}$

It has various merits like: Compatibility with X-ray, magnetic resonance imaging (MRI), and computed tomography (CT) imaging without producing artifacts, High 
compressive strength, Natural color for excellent aesthetics, Ease of chairside preparation and modification by dentists.

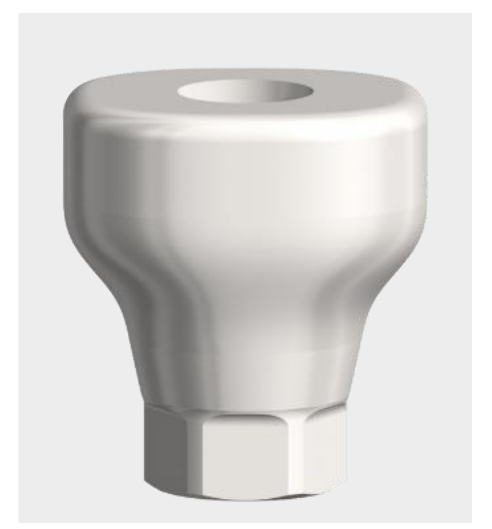

Fig. 4: Peek healing abutments

\section{Abutment emergence profile: How it changes the soft-tissue position}

The emergence profile is the portion of the abutment from the head of the implant to the free gingival margin, and it has significant impact on the shape and position of the soft tissue. Generally, a larger diameter emergence profile compresses the soft tissue and move it apically, while a narrowed emergence profile will allow the soft tissue to drape in a more coronal position neither design is indicated for all situations, and the design must be based on the needs of the particular clinical scenario. Ultimately, the emergence profile design must be tested in-situ, and time must be allotted for the patient's peri-implant tissues to respond and mature before further alterations are made. The maturation process should be allowed to take place for at least 4 weeks. ${ }^{19}$

Repeated disconnection of components from the implants results in significant loss of peri-implant bone and soft tissue ( $\sim 0.7 \mathrm{~mm}$ and $1.5 \mathrm{~mm}$, respectively). ${ }^{20}$ This loss is most acutely seen during the initial osseointegration and soft-tissue maturation stage. Thus, repeated removal of the abutment and reshaping of the emergence profile should be kept to an absolute minimum and postponed at least 4 months following implant placement.

Any enlargement of the emergence profile will result in blanching of the soft tissues. This is caused by a transient decrease in blood flow due to pressure from the abutment. In the subsequent weeks, the peri-implant tissues will reorganize and adjust to the pressure by settling at a more apical position. The speed and extent to which this happens depends on the amount of increased abutment size, the tissue thick-ness, oral hygiene, and bone position. Thus, it is advisable that critical changes in soft-tissue contours be performed in a provisional stage, allowing the tissue to mature and adjust for at least 4 weeks and then re-evaluating before making the final impression. Special attention should be given to the shape and apparent width of the tooth at the cervical area. The mesio-distal width of the abutment at this position should mirror that of the contralateral tooth or teeth. Within the peri-implant zone, the most delicate and challenging aspect is the management of the papilla. This small, pyramid-shaped soft tissue depends on its attachment to adjacent natural tooth roots and underlying bone for its height and blood supply. Adjacent to an implant, at least half of this support no longer exists. Attempts have been made (with occasional success) in compressing the papilla from the sides to increase its height. ${ }^{21}$ However, this is an unpredictable approach, and greater success may be achieved by al-lowing space (open gingival embrasure) during the provisional stage for the papilla to mature to its maximum vertical length and then closing any remaining open embrasure with the definitive prostheses by extending the interproximal contact point apically.

\section{Conclusion}

A major challenge for clinicians is to understand the biologic response to each material, as well as the best indication for using each of the different types while selecting implant abutments. An Ideal restorative prosthesis can be fabricated by understanding abutment classification, types of material used and the desires of the patients. The treatment, however remains the same and will invariably involve correct treatment planning, decision making and sequencing of treatment that will ensure a successful end result. Custom implant abutments allow the clinician to improve an implant's emergence profile, customize cervical margins so that they are anatomically shaped in accordance to the natural tooth root, and compensate for poor implant angulation. All of these are essential for optimum esthetic outcomes.

\section{Source of funding}

None.

\section{Conflict of interest}

None.

\section{References}

1. Misch CE, Screw-retained versus cement-retained implantsupported prostheses. Pract Periodontics Aesthet Dent 1995;7(9):15-8.

2. Karunagaran, Sanjay \& Paprocki, Gregory \& Wicks, Russell \& Markose, Sony. A review of implant abutments--abutment classification to aid prosthetic selection. J Tennessee Dent Assoc 2014;93:18-23.

3. Priest G. Virtual-designed and computer-milled implant abutments. J Oral Maxillofac Surg 2005;63(9 Suppl 2):22-32.

4. Rekow ED. CAD/CAM in dentistry: A historical perspective and view of the future. JCDA 1992;58:283.

5. Mehl A, Hickel R. Current state of development and perspectives of machine-base production methods for dental restorations. Int J Comput Dent 1999;2:9.

6. Osario J. Use of the Atlantis Abutment in restorative practice speeds time to function and aesthetics. Dental Implantol Update 2000;11:57.

7. Kerstein RB, Castellucci F, Osorio J. Ideal gingival form with computer-generated permanent healing abutments. Compendium 2000;21:793.

8. Kerstein RB, Osorio J. Utilizing computer-generated duplicate titanium custom abutments to facilitate intraoral and laboratory implant prosthesis fabrication. Pract Proced Aesthet Dent 2003; 15:311. 
9. Ganz SD: Computer-milled patient-specific abutments: Incredible quality with unprecedented simplicity. Pract Proced Aesthet Dent 2003;15:37(suppl 8).

10. Andersson M, Oden A: A new all-ceramic crown: Dense-sintered, high-purity alumina coping with porcelain. Acta Odont Scand 1993;51:59.

11. Van Dooren E. Restoring implants with Procera technology and optimal tissue management. Pract Proced Aesthet Dent 15:23, 2003 (suppl 8).

12. Henriksson K, Jemt T: Evaluation of custom-made Procera ceramic abutments for single-implant tooth replacement: A prospective 1-year follow-up study. Int J Prosthodont 2003;16:626.

13. Drago CJ, Two new clinical/laboratory protocols for CAD/CAM implant restorations. J Am Dent Assoc 2006;137(6):794-800.

14. Kapos T. Computer- aided design and computer- assisted manufacturing in prosthetic implant dentistry. Int J Oral Maxillofac Implants 2009;24 Suppl:110-7.

15. Implant Abutment Materials Clinical and Laboratory Manual of Dental Implant Abutments, First Edition. Edited by Hamid R. Shafie. ( 2014 John Wiley \& Sons, Inc. Published 2014 by John Wiley \& Sons, Inc.

16. Williams DF, McNamara A, Turner RM. Potential of polyetheretherketone (PEEK) and carbon- fibre-reinforced PEEK in medical applications. J Mater Sci Lett 1987;6:188190.

17. Hahnel S, Wieser A, Lang R, Rosentritt M. Biofilm formation on the surface of modern implant abutment materials. Clin Oral Implants Res 2014 [Epub ahead of print].

18. Koutouzis T, Richardson J, Lundgren T. Comparative soft and hard tissue responses to titanium and polymer healing abutments. J Oral Implantol 2011;37:174-82.

19. Schoenbaum, Todd. (2015). Abutment Emergence Profile and Its Effect on Peri-Implant Tissues. Compendium of continuing education in dentistry (Jamesburg, N.J. 1995;36:474-9.

20. Abrahamssoni, Berglundh T, Lindhe J. The mucosal barrier following abutment dis/reconnection. J Clin Periodontol 1997;24(8):568- 572.

21. Lesage BP. Improving implant aesthetics: prosthetically generated papilla through tissue modeling with composite. Pract Proced Aesthet Dent 2006;18(4):257-63.

How to cite this article: Sagar M, Vishnoi L, Kumar V, Aeran H. Concernment of various abutments for enhancing emergence profile in implant prosthodontics. Int J Oral Health Dent 2019;5(4):174-9. 